\title{
Traffic Signal Controller for Mixed Traffic Conditions
}

\author{
Budi Yulianto ${ }^{1}$, Setiono ${ }^{2}$ \\ ${ }_{1,2}^{2}$ (Civil Engineering Department, Sebelas Maret University, Indonesia)
}

\begin{abstract}
Fuzzy logic has been widely used to develop an adaptive traffic signal controller because it allows qualitative modelling of complex systems. However, existing research has developed Fuzzy Logic Traffic Signal Controller (FLTSC) based on non-mixed traffic conditions. These FLTSC are not appropriate to the mixed traffic conditions of developing countries where the traffic streams consist of different types of vehicles with a wide variation in their static, dynamic and operating characteristics.

This paper describes the design and evaluation of an adaptive traffic signal controller based on fuzzy logic for an isolated four-way intersection with specific reference to mixed traffic in developing countries. The controller is designed to be responsive to real-time traffic demands. Video image processing has been proposed to capture traffic data such as maximum queue length (in metres) and average occupancy rate (in \%) from each approach of the intersection. The proposed FLTSC uses maximum queue lengths and average occupancy rates collected during the previous cycle in order to estimate the number of seconds of green time required by each set of signal groups (stage) during the next cycle.

The effectiveness of the proposed FLTSC was examined and analysed by the simulation program VISSIM. The performance of this controller is to be contrasted with the Fixed Time Controller (FTC) and Vehicle Actuated Controller - Extension Principle (VAC-EP) for different traffic conditions on a simulated fourway intersection. The simulation results indicate that the performance of the proposed FLTSC is generally better than the FTC and VAC-EP, especially in cases time-varying traffic.
\end{abstract}

Keywords: fuzzy logic, mixed traffic, signal control

\section{Introduction}

Traffic signal control is a measure that is commonly used at road intersections to minimise vehicular delays. Traffic signal control at road intersections allows vehicle movements to be controlled by allocating time intervals, during which separate traffic demands from each approach to the intersection can make use of the available road space. There are three traffic signal control types for isolated intersections [1], namely Fixed Time control, Vehicle Actuated control and Self Optimising control. Under Fixed Time (FT) control, all signal timing parameters are pre-computed and kept constant. These parameters are calculated based on historical traffic data. This method usually shows good results in normal traffic conditions, but sometimes FT controls fail to cope with complex, time varying traffic conditions [2, 3, 4 and 5].

Vehicle Actuated (VA) control represents an improvement over FT control. VA control has the ability to extend the length of green time for a particular stage, based on traffic demand as registered by vehicle actuated detectors on the intersection approaches. However, this method has a disadvantage in that the extension of green is determined by traffic demand from the stage having right of way, without considering traffic demand from the stages having red [6]. This leads to extending the green stage inefficiently, particularly when there are long queues waiting at red signals. In general, VA control performance deteriorates with heavy traffic conditions [7].

Self Optimising or Adaptive Traffic Signal (ATS) control is designed to address those deficiencies. ATS control adjusts the length of green time for a particular stage, based both on traffic demand from the stages having right of way, and having red. The algorithm of the controller is usually developed using a complex conventional mathematical model. A number of approaches have been proposed for the design and implementation of ATS control systems, such as TOL (8) and MOVA [9]. Due to the complexity of finding the optimum signal setting parameters using conventional mathematical models, researchers have applied artificial intelligence techniques to address the problems.

Fuzzy logic is one of the artificial intelligence techniques shown to offer a very promising mathematical approach to modelling traffic and transportation processes which are characterised by subjectivity, uncertainty and imprecision. It was first suggested by Zadeh [10]. The first work on the application of fuzzy logic to traffic signal control was done by Pappis and Mamdani [11]. Since then, fuzzy logic has been used widely to develop an ATS controller that is both theoretical and practical, because it is adequate for qualitative modelling of complex systems, which are not easy to solve using conventional mathematic models [3,12 and 13] and the controller uses the fuzzy rule base (i.e. 'IF - THEN' rules), which is easy to develop and modify (14), instead of using complex conventional mathematical models. Research on the application of the fuzzy 
logic to traffic signal control has generally reported better performance from the Fuzzy Logic Traffic Signal Controller (FLTSC) when compared to the FTC and certain VA controllers [3, 4, 5, 7, 11, and 13].

Traffic control in isolated intersections in Indonesia is done with FT signals. FT control is not suitable for intersections where the traffic demand fluctuation is high, and demand responsive traffic signal control must be introduced to reduce traffic congestion. However, all existing demand responsive traffic signal control such as VA and ATS controls (including FLTSC) has been developed for non-mixed traffic conditions (i.e. in developed countries), considering only motor vehicles in clearly defined lanes, neglecting motorcycles. This is quite different to mixed traffic conditions (i.e. in developing countries), where traffic composition in the roadway is heterogeneous, consisting of different vehicle types with widely varied static, dynamic and operating characteristics, and with a particularly high proportion (30\% - 70\%) of motorcycles. All types of vehicle share in, and compete together for, the same road space at intersections. This combined flow of all vehicle types generates different vehicular interaction and stream flow behaviour. Due to lack of lane discipline, queues at intersections are based on optimum road space utilization, which means vehicles may occupy any position across the road based on the space available. Figure 1 shows queue is built up during red signal in mixed traffic conditions.

It is obvious that traffic behaviour in mixed traffic conditions is different from that in non-mixed traffic conditions. Hence, there is a need for a new ATS controller that can operate at isolated intersections taking account of the mixed traffic in developing countries. The main objective of this research is to develop an ATS controller based on fuzzy logic for an isolated intersection, with reference to the mixed traffic in developing countries, including a high proportion of motorcycles. Simulations are used to examine and analyse the effectiveness of the proposed FLTSC. Then, the performance of an isolated intersection under the control of the proposed FLTSC is contrasted to that under FT and VA controls. The performance criteria used for comparison are the average delay of vehicles.
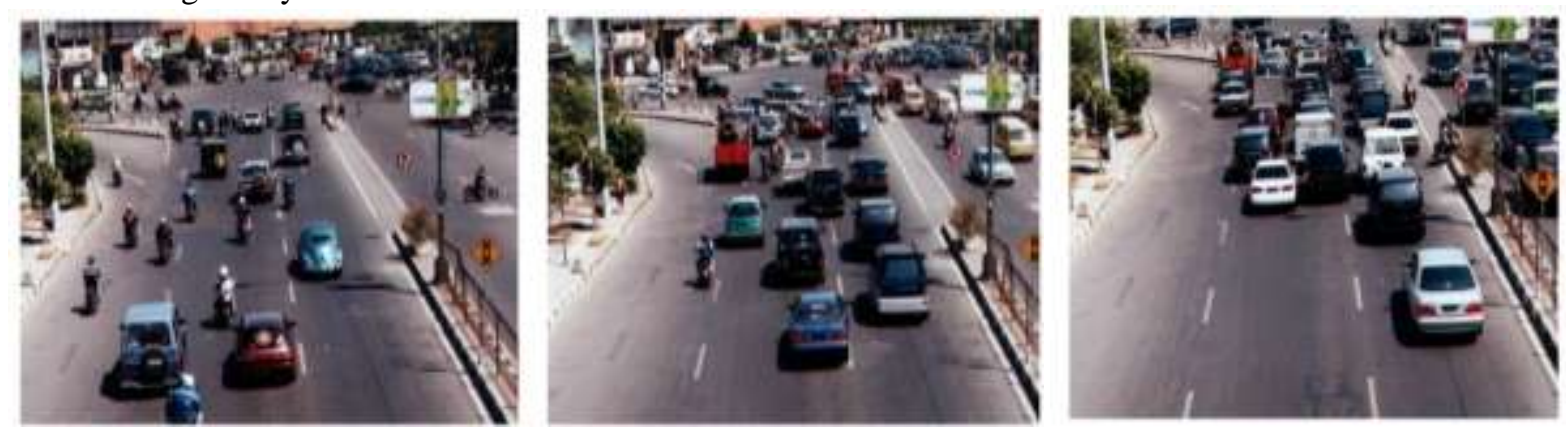

Fig 1 . The queue is built up during red signal in mixed traffic conditions

\section{Overview Of Traffic Signal Control Strategies}

\subsection{Fixed Time Control}

FT control uses a preset cycle and green times to change the signals. The design of a signal timing plan in Indonesia is carried out using techniques set out in the Indonesian Highway Capacity Manual [15]. The equations used to calculate the cycle length and green time were adapted from the Webster [16] model. The cycle length (seconds), according to the Indonesian Highway Capacity Manual (IHCM), is calculated by:

$$
C=\frac{1.5 L T I+5}{1-\sum F R_{C R I T}}
$$

where
LTI
is the lost time per cycle (seconds)
$F R \quad$ is ratio of flow to saturation flow $(\mathrm{Q} / \mathrm{S})$
FRCRITn is the maximum value of FR among the signal group for the same stage
EFRCRIT is the summation of FRCRITn for all $\mathrm{N}$ stages in the cycle

$$
\Sigma F R_{C R I T}=\sum_{n=1}^{N} F R_{C R I T} n
$$

The green time of nth stage is calculated by: 


$$
g_{n}=(C-L T I) \frac{F R_{C R I T n}}{\sum F R_{C R I T}}
$$

\subsection{Vehicle Actuated Control}

The VA control Extension Principle (VAC-EP) has been used widely at isolated intersection in many countries, especially in the United States of America [17]. Under this control, the green time of the phase is adjusted based on the extension time and time gap between vehicles crossing the point detector a certain distance from the stop line (D metres). A typical detector configuration of the Extension Principle at an isolated intersection is presented in Figure 2.

There are three parameters required by the VA control Extension Principle, namely the minimum green time, the extension time and the maximum green time. A green signal is activated for at least the minimum green time to provide sufficient time for all vehicles potentially stored between the detector and the stop line to enter the intersection. The green interval is extended by resetting the extension time every time an actuation vehicle is recorded after the minimum green time expires. If the detectors record another vehicle within this extension time, the green will be extended again from the time of this actuation, by the length of the extension time. If the time gap (headway) between vehicles becomes greater than this extension time, then this green interval will be terminated before it reaches its maximum green value. In such circumstances, where there are no vehicles detected on a particular approach, the controller can skip over that stage and move directly to the next stage in the sequence.

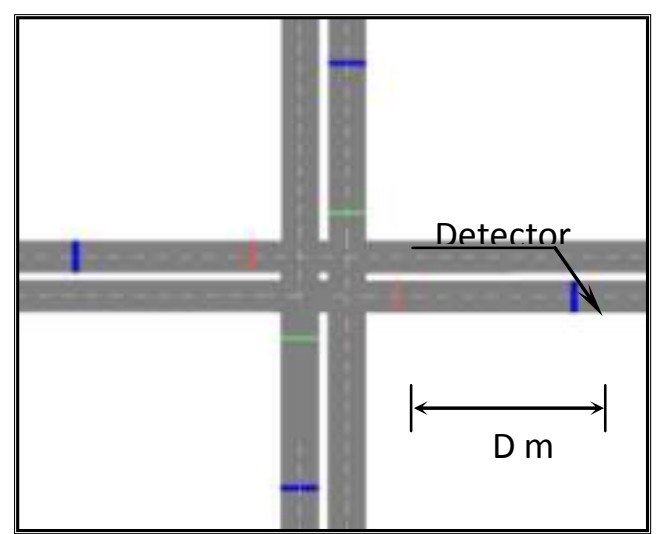

Fig 2. Typical detector configuration of Extension Principle at an isolated intersection

\section{Fuzzy Logic Traffic Signal Controller}

\subsection{Design Criteria And Constraints}

In designing the proposed FLTSC system the following criteria are defined:

1. The intersection is an isolated four-way intersection with traffic coming from West, East, North and South (see Figure 2).

2. The signal has two stages. Stage 1 consists of traffic from East and West (E-W); and stage 2 consists of traffic from North and South (N-S).

\subsection{Structure Of The Proposed Fuzzy Logic Traffic Signal Controller And Traffic Flow Modelling}

The structure of the proposed FLTSC system is illustrated in Figure 3. During the simulation period, the traffic flow model simulates traffic operation and every second it produces traffic information through vehicle detectors. This information is used as a crisp input for the fuzzy logic module at the end of the cycle. The fuzzy logic module consists of three parts, namely fuzzification, fuzzy inference and defuzzification. The crisp output from the fuzzy logic module is used by the signal controller program to determine the signal status for the following cycle, and returns this information to the traffic flow model. At the end of the simulation period, output values (i.e. measures of performance) will be obtained, namely the average delay of vehicles. This process is carried out in the simulation package VISSIM.

As an adaptive traffic signal controller, the proposed FLTSC needs vehicular detectors to provide accurate information of the prevailing traffic conditions in real time. This information is used as input data for the controller.

In this research, advanced video image processing (VIP) was used to collect traffic parameters. 


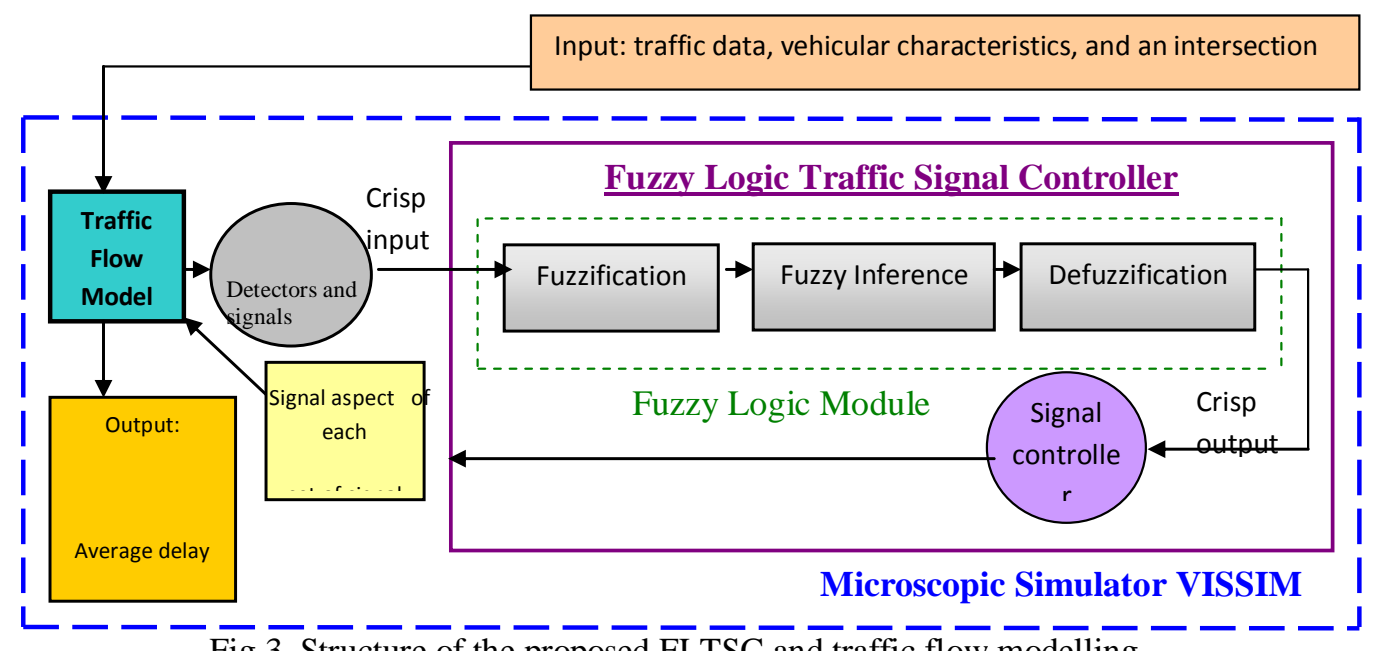

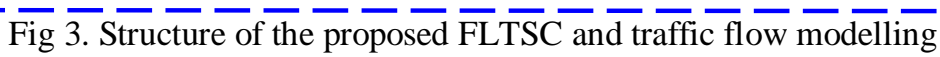

In order to derive useful information from the detector data, it is important to be selective and to use data that can give meaningful information about actual traffic flow. Factors which influence the data selected include the setting of the signal group, (i.e. red or green) and the placement of the detection area [18]. The proposed FLTSC uses two input fuzzy variables as follows:

1. Maximum Queue Length (metres). Queue length is defined as the distance in metres from the stop line over which vehicles are queueing [19]. During the red period, the VIP records the longest tail of queue (i.e. maximum queue length) irrespective of the lane in which it occurs, every second. Then the maximum queue length at the onset of green periods is used as an input.

2. Average Occupancy Rate $(\%)$. The detection area is placed one metre downstream of the stop line of each approach, to record occupancy rate. During the green period, the VIP records the occupancy rate every second and the average occupancy rate for that period is used as an input.

The output fuzzy variable of the proposed FLTSC is Weight. Weight (a value between 0 and 100) is an indicator of the degree of need that the signal group (SG) requires green. For example if the weight of $\mathrm{SG}_{1}$ is 75 and that of $\mathrm{SG}_{2}$ is 25 , it means that $\mathrm{SG}_{1}$ needs green more than $\mathrm{SG}_{2}$.

\subsection{Fuzzy Control Strategy}

Figure 4 shows the process of calculation of new green time values for the next cycle, using traffic data from the previous cycle. The proposed FLTSC uses traffic information about maximum queue lengths and average occupancy rates collected during the previous cycle, in order to estimate the number of seconds of green time required by each set of signal groups (stage) during the next cycle.

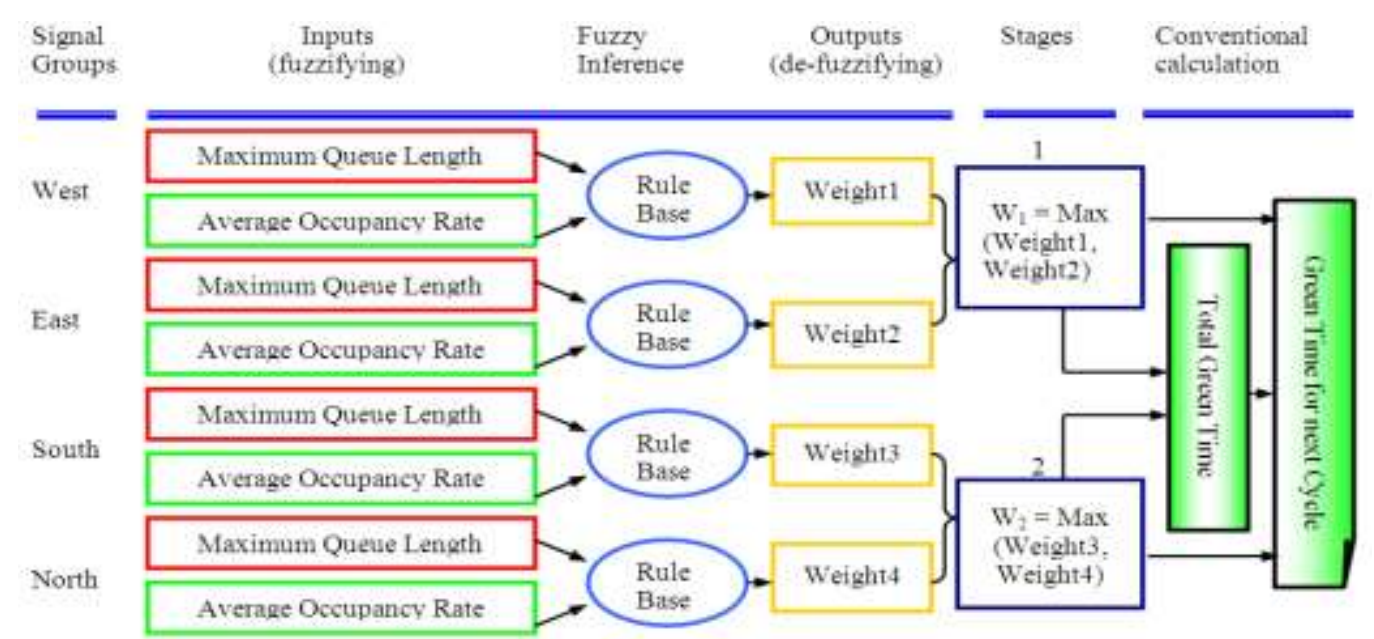

Fig 4. Calculation of new green time value for the next cycle using traffic data from the previous cycle

The fuzzy logic module uses the input fuzzy variables maximum queue length and average occupancy rate collected during the previous cycle, to derive weight value of each SG using the fuzzy rule base. The maximum weight value between SGs West and East is chosen as the weight value for stage $1\left(\mathrm{~W}_{1}\right)$. This method 
is applied for stage $2\left(\mathrm{~W}_{2}\right)$ as well. Then weight values of stage 1 and stage 2 are used to calculate the total green time in a cycle. The weight values of stages and the total green time in a cycle are used to estimate the duration of green time that stages require in the next cycle. Both total green time and green time of each stage are calculated using conventional calculation. The calculation of total green time is shown in Equation 4.

$$
\text { Total_GT }=\left(\sum W_{n}-\operatorname{Min} 1\right) *\left[\frac{\operatorname{Max} 2-\operatorname{Min} 2}{\operatorname{Max} 1-\operatorname{Min} 1}\right]+\operatorname{Min} 2
$$

Then, the green time of each stage is calculated by Equation 5 .

where:

$$
G T \_ \text {Stage }{ }_{n}=\frac{W_{n}^{*} \text { Total_GT }}{\sum W_{n}}
$$

$$
\Sigma W_{n} \quad \text { is total weight of stages }
$$

$\operatorname{Min} 1$ and Max 1 are minimum and maximum value of total weight, namely 0 and 200 respectively

Min2 and Max2 are minimum and maximum value of total green time in a cycle

$n$

is stage index

\subsection{Fuzzification And Membership Functions}

In this research, the triangular and trapezoidal types of membership function are used because of their computational simplicity and efficiency. The number of fuzzy partitions for the input and output linguistic variables is determined by a common sense, trial and error method [20 and 21]. Membership functions of each input and output fuzzy variable of the proposed FLTSC are as follows: Maximum Queue Length and Average Occupancy Rate have \{Low, Medium, High and Very High\} and Weight has \{Very Very Low, Very Low, Low, Medium, High, Very High and Very Very High \} linguistic labels, respectively. The graphical representation of the membership functions of these linguistic variables is shown in Figure 5. The configuration of these membership functions is done according to expert observation of the system; the membership functions are tuned by studying the performance of the controller under various traffic conditions, to improve the performance of the controller [7 and 18].

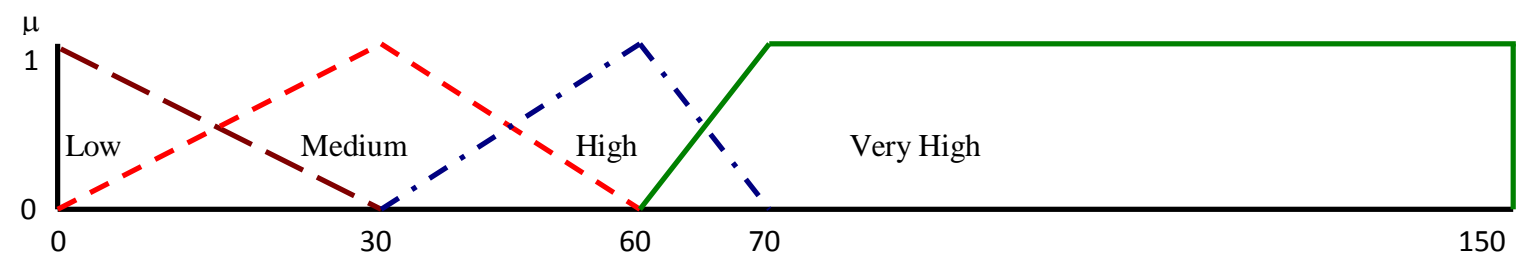

(a). Input Fuzzy Variable 1: Maximum Queue Length (metres)

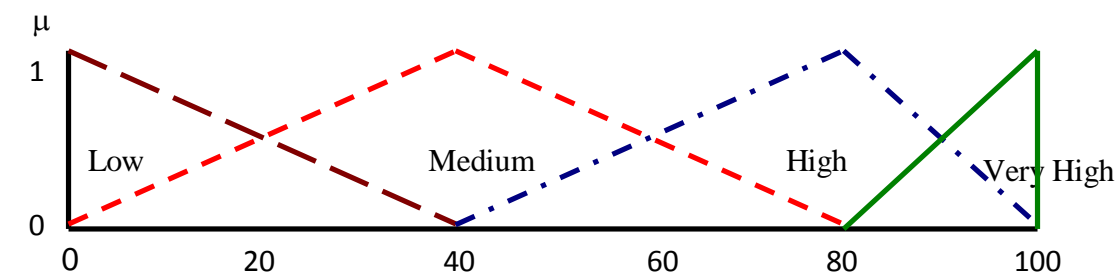

(b). Input Fuzzy Variable 2: Average Occupancy Rate (\%)

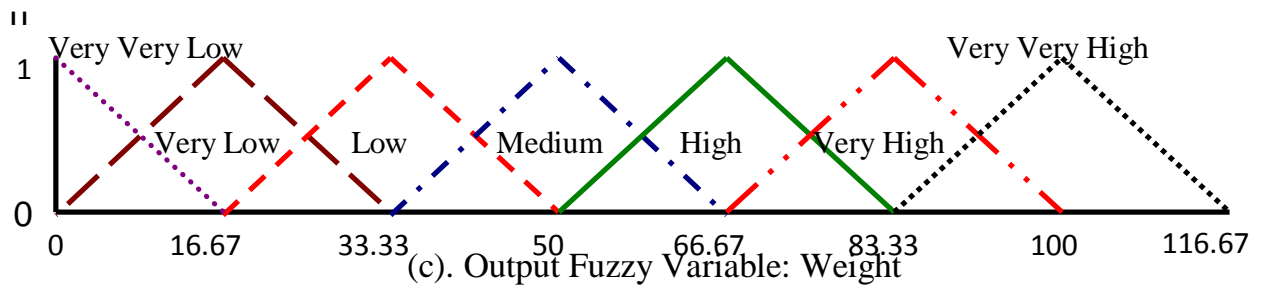

Fig 5. Graphical representation of membership functions of the proposed FLTSC 


\subsection{Fuzzy Rule Base}

The basic function of the fuzzy rule base is to represent expert knowledge in a form of If-Then rule structure. The fuzzy rule base is a set of fuzzy rules. It maps the combination of fuzzy inputs to the fuzzy output. The number of rules is equal to the number of input combinations derived from the number of membership functions per input [22]. The proposed FLTSC has two inputs, each having four membership functions; so the number of fuzzy rules will equal sixteen.

In this research, the fuzzy rule base was adjusted based on the trial and error method [11]. Using trial and error, it has been found that the input fuzzy variable maximum queue length is more sensitive to the performance of the controller than the average occupancy rate. Therefore, the fuzzy rule base was designed to give more priority to the input fuzzy variable maximum queue length. The configuration of the fuzzy rules in the matrix structure of the proposed FLTSC is shown in Figure 6. An example of fuzzy rule 9 is:

IF Max. Queue length is High AND Avg. Occupancy rate is Low THEN Weight is Medium

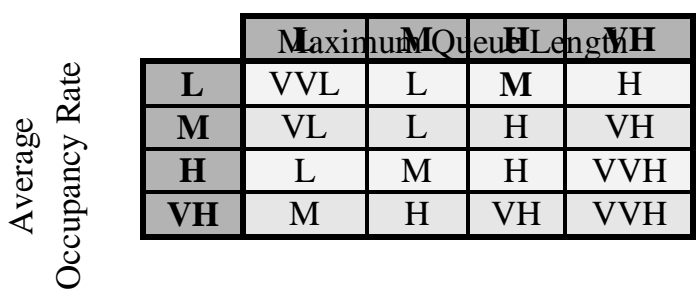

$$
\begin{array}{ll}
\text { Note: } & \\
\boldsymbol{V} \boldsymbol{V} \boldsymbol{L} & =\text { Very Very Low } \\
\mathrm{VL} & =\text { Very Low } \\
\mathrm{L} & =\text { Low } \\
\mathrm{M} & =\text { Medium } \\
\mathrm{H} & =\text { High } \\
\mathrm{VH} & =\text { Very High }
\end{array}
$$

Fig 6. Fuzzy rule base in matrix structure to derive a weight value for each signal group

\subsection{Fuzzy Inference Engine And Defuzzification}

Fuzzy inference evaluates the control rules stored in the fuzzy rule base. The fuzzy inference system used in this research is the Mamdani and Assilian [23] method, because of its computational simplicity and efficiency. Defuzzification is a process to convert the fuzzy output values of a fuzzy inference to crisp output values. According to Driankov et al [22], the Centre-of-Sum $(\mathrm{CoS})$ defuzzification method gives better performance in term of continuity, disambiguity, plausibility, computer complexity and weight counting. In this research, therefore, the CoS method was used to defuzzify the fuzzy output variable weight.

\subsection{Simulation Tool - VISSIM And VAP Language}

In these simulation studies, VISSIM 4.10 was used to simulate traffic and examine the effectiveness of the proposed FLTSC. In order to simulate mixed traffic conditions, all vehicle types were modelled individually. The vehicle can occupy any position across the available lane space depend on the safe lateral clearance among vehicles. Motorcycles use inter-vehicular spaces to come to the front of the queue, and fill any lane space available between two vehicles. A motorcycle can squeeze between two vehicles moving side-by-side, or two successive vehicles moving in the same lane.

A new approach used in this research was that both the fuzzy logic module and signal controller program were developed using the VAP language [24]. This way, the signal controller program could interact directly with VISSIM via an external signal state generator. This approach offers less computational complexity than if the fuzzy logic module were developed using a commercial fuzzy logic toolbox (e.g. SIEFUZZY, MATLAB) or using $\mathrm{C} / \mathrm{C}++$ programming language, which would need several interfaces.

\subsection{Case Study}

\section{Simulation Studies}

In order to evaluate the effectiveness of the controllers, four case studies with different traffic compositions and volumes were carried out, namely:

Case 1: Traffic flow is constant during a one-hour period

Case 2: Traffic flow varies, every 15 minutes the traffic changes.

Case 3: Similar to case 2, but the traffic composition is different.

Case 4: Traffic flow used is based on real data from the Sutomo-Diponegoro signalised intersection in the city of Surabaya, Indonesia.

Traffic composition and set of traffic volume for all case studies are presented in Tables 1 and 2. 
Table 1. Traffic composition (\%) used in the simulation for cases 1, 2, 3 and 4.

\begin{tabular}{|l|c|r|r|r|r|r|}
\hline Case & MC & CAR & LGV & MGV & HGV & BUS \\
\hline $1 \& 2$ & 40.0 & 45.0 & 6.0 & 4.0 & 2.5 & 2.5 \\
\hline 3 & 30.0 & 40.0 & 10.0 & 5.0 & 5.0 & 10.0 \\
\hline 4 & 47.2 & 46.5 & 3.9 & 1.4 & 0.2 & 0.8 \\
\hline
\end{tabular}

Table 2. Set of traffic volumes (vehicles/hour) used in the simulation for cases 1, 2, 3 and 4.

\begin{tabular}{|c|c|c|c|c|c|c|c|c|}
\hline \multirow[t]{2}{*}{ Case } & \multicolumn{2}{|c|}{ Traffic Volumes } & \multirow[t]{2}{*}{ Case } & \multicolumn{2}{|c|}{ Traffic Volumes } & \multirow[t]{2}{*}{ Case } & \multicolumn{2}{|c|}{ Traffic Volumes } \\
\hline & E-W & $\mathrm{N}-\mathrm{S}$ & & E-W & $\mathrm{N}-\mathrm{S}$ & & E-W & $\mathrm{N}-\mathrm{S}$ \\
\hline $1 \mathrm{a} ; 2 \mathrm{a}$ & 1080 & 1080 & $1 \mathrm{~g} ; 2 \mathrm{~g}$ & 1440 & 1800 & $3 b$ & 1080 & 1440 \\
\hline $1 b ; 2 b$ & 1080 & 1440 & $1 \mathrm{~h} ; 2 \mathrm{~h}$ & 1440 & 2160 & $3 c$ & 1080 & 1800 \\
\hline $1 \mathrm{c} ; 2 \mathrm{c}$ & 1080 & 1800 & $1 \mathrm{i} ; 2 \mathrm{i}$ & 1440 & 2520 & $3 d$ & 1440 & 1440 \\
\hline $1 d: 2 d$ & 1080 & 2160 & $1 \mathrm{j} ; 2 \mathrm{j}$ & 1800 & 1800 & $3 \mathrm{e}$ & 1440 & 1800 \\
\hline $1 \mathrm{e} ; 2 \mathrm{e}$ & 1080 & 2520 & $1 \mathrm{k} ; 2 \mathrm{k}$ & 1800 & 2160 & 4 & $1935(\mathrm{E})$ & $2071(\mathrm{~N})$ \\
\hline $1 \mathrm{f} ; 2 \mathrm{f}$ & 1440 & 1440 & $3 a$ & 1080 & 1080 & & $2031(\mathrm{~W})$ & $1848(\mathrm{~S})$ \\
\hline
\end{tabular}

\subsection{Parameter Controllers}

In the case of the FTC, signal timing remains fixed during the simulation time. The signal timing for the FTC was calculated using the IHCM method. The cycle time and green time were calculated using the Equations 1 and 3, respectively. Table 3 shows signal timing used for the FTC. One of the most critical parameters that affect the overall performance of VAC-EP is the extension time. The extension time used in this study based on the results of the simulation studies carried out by Yulianto [25]. His simulation studies showed that the VAC-EP with detector position of 30 metres and extension time of 3.0 seconds gave the best controller performance (in term of average delay) in mixed traffic conditions. The minimum green time of VAC-EP for each phase was 7.0 seconds. The maximum green time of VAC-EP for each phase was determined by calculating the green time at FTC and multiplying by a factor of 1.5 [26].

Table 3. Cycle time and green time used for the FTC

\begin{tabular}{|c|c|c|c|c|c|c|c|}
\hline \multirow[t]{2}{*}{ Case } & \multicolumn{2}{|c|}{ Green Time (second) } & \multirow{2}{*}{$\begin{array}{c}\text { Cycle Time } \\
\text { (second) }\end{array}$} & \multirow[t]{2}{*}{ Case } & \multicolumn{2}{|c|}{ Green Time (second) } & \multirow{2}{*}{$\begin{array}{c}\text { Cycle Time } \\
\text { (second) }\end{array}$} \\
\hline & $E-W$ & $\mathrm{~N}-\mathrm{S}$ & & & $E-W$ & $\mathrm{~N}-\mathrm{S}$ & \\
\hline $1 \mathrm{a} ; 2 \mathrm{a}$ & 10 & 10 & 28 & $1 \mathrm{j} ; 2 \mathrm{j}$ & 22 & 22 & 52 \\
\hline $1 b ; 2 b$ & 10 & 14 & 32 & $\begin{array}{l}1 \mathrm{k} ; \\
2 \mathrm{k}\end{array}$ & 26 & 31 & 65 \\
\hline $1 \mathrm{c} ; 2 \mathrm{c}$ & 11 & 18 & 37 & $3 a$ & 12 & 12 & 32 \\
\hline $1 d ; 2 d$ & 12 & 23 & 43 & $3 b$ & 12 & 17 & 37 \\
\hline $1 \mathrm{e} ; 2 \mathrm{e}$ & 13 & 31 & 52 & $3 c$ & 14 & 23 & 45 \\
\hline $1 \mathrm{f} ; 2 \mathrm{f}$ & 15 & 15 & 38 & $3 d$ & 19 & 19 & 46 \\
\hline $1 \mathrm{~g} ; 2 \mathrm{~g}$ & 16 & 19 & 43 & $3 e$ & 22 & 27 & 57 \\
\hline $1 \mathrm{~h} ; 2 \mathrm{~h}$ & 18 & 27 & 53 & 4 & 24 & 23 & 55 \\
\hline $1 \mathrm{i} ; 2 \mathrm{i}$ & 21 & 36 & 65 & & & & \\
\hline
\end{tabular}

In the proposed FLTSC, minimum and maximum values of total green time in a cycle are imposed to avoid extremely long and short cycle length. These values determine the upper and lower limits of the cycle length. The minimum and maximum values of total green time in a cycle for the proposed FLTSC are 10 seconds and 62 seconds, respectively. The configuration of the membership functions of input/output fuzzy variables and fuzzy rule base as shown in Figures 4 and 5 were same for all cases study. For all controllers, the amber and all red periods are the same. Amber and all red periods are 3 seconds and 1 second respectively.

\subsection{Simulation Results}

Identical simulations were run for approximately one-hour periods on the FTC, VAC-EP and proposed FLTSC to produce the output value (i.e. delay) of vehicles, for all case studies. The output value of the proposed FLTSC was then compared with the FTC and VAC-EP. Comparison of the average delay of vehicles at the intersection in one-hour simulation time between the FTC, VAC-EP and proposed FLTSC for cases 1, 2, 3 and 4 can be seen in Table 4.

The simulation results for case 1 show that generally the average delay of the proposed FLTSC is close to the average delay of the FTC. The simulation results for case 2 indicate that the proposed FLTSC performs much better than the FTC for all different set traffic volumes. The proposed FLTSC yields an improvement of 
2.7-39.5\% on the average delay. The simulation results for case 3 reveal that the proposed FLTSC outperforms the FTC for all different set of traffic volumes. The average delays savings by the proposed FLTSC are 9.3$35.4 \%$. The simulation results for case 4 show that the proposed FLTSC has slightly more average delay than the FTC. The average delay of the proposed FLTSC is $1.4 \%$ higher than the average delay of the FTC.

The simulation results for case 1 show that in general the VAC-EP and proposed FLTSC produce little difference in terms of the average delay of vehicles at the intersection in one-hour simulation time. The performance of the proposed FLTSC reduced as the traffic flows of North-South approaches increased.

The simulations results for case 2 indicate that the proposed FLTSC yields less average delay than the VAC-EP in all cases, apart from cases $2 \mathrm{e}, 2 \mathrm{~h}$ and $2 \mathrm{j}$. In case 2 , except cases $2 \mathrm{e}, 2 \mathrm{~h}$ and $2 \mathrm{j}$, the proposed FLTSC reduce the average delay by 3.3-22.6\% against the VAC-EP. In cases $2 \mathrm{e}, 2 \mathrm{~h}$ and $2 \mathrm{j}$, the proposed FLTSC show $1.7 \%, 3.5 \%$ and $7.5 \%$ increase in the average delay, respectively against the VAC-EP.

Table 4. Average delay of vehicles at the intersection in one-hour simulation time of the FTC, VAC-EP and proposed FLTSC for cases 1, 2, 3 and 4

\begin{tabular}{|c|c|c|c|c|c|}
\hline \multirow{2}{*}{ Cases } & \multicolumn{5}{|c|}{ Average Delay (seconds) } \\
\hline & FTC & FLTSC & Improvement (\%) & VAC-EP & Improvement (\%) \\
\hline $1 \mathrm{a}$ & 11.3 & 11.4 & $-0.6 \%$ & 11.1 & $-2.0 \%$ \\
\hline $1 \mathrm{~b}$ & 13.1 & 13.0 & $0.9 \%$ & 13.5 & $3.7 \%$ \\
\hline $1 \mathrm{c}$ & 15.5 & 16.0 & $-3.1 \%$ & 15.3 & $-4.6 \%$ \\
\hline $1 d$ & 17.7 & 19.6 & $-10.8 \%$ & 18.0 & $-9.0 \%$ \\
\hline $1 \mathrm{e}$ & 26.6 & 26.8 & $-0.8 \%$ & 22.1 & $-21.3 \%$ \\
\hline $1 \mathrm{f}$ & 15.1 & 15.8 & $-4.7 \%$ & 15.6 & $-1.6 \%$ \\
\hline $1 \mathrm{~g}$ & 18.7 & 18.5 & $1.1 \%$ & 18.6 & $0.9 \%$ \\
\hline $1 \mathrm{~h}$ & 22.4 & 23.8 & $-5.9 \%$ & 23.2 & $-2.6 \%$ \\
\hline $1 \mathrm{i}$ & 31.3 & 33.5 & $-7.2 \%$ & 32.6 & $-2.7 \%$ \\
\hline $1 \mathrm{j}$ & 22.6 & 23.0 & $-1.9 \%$ & 22.4 & $-2.8 \%$ \\
\hline $1 \mathrm{k}$ & 31.4 & 31.5 & $-0.1 \%$ & 32.1 & $1.9 \%$ \\
\hline $2 a$ & 21.3 & 13.1 & $38.3 \%$ & 16.1 & $18.6 \%$ \\
\hline $2 b$ & 32.5 & 19.6 & $39.5 \%$ & 23.5 & $16.3 \%$ \\
\hline $2 c$ & 28.8 & 19.4 & $32.4 \%$ & 25.1 & $22.6 \%$ \\
\hline $2 d$ & 30.8 & 27.3 & $11.2 \%$ & 29.7 & $7.9 \%$ \\
\hline $2 e$ & 36.0 & 35.1 & $2.7 \%$ & 34.5 & $-1.7 \%$ \\
\hline $2 f$ & 31.1 & 18.7 & $39.9 \%$ & 23.1 & $18.8 \%$ \\
\hline $2 g$ & 32.2 & 27.0 & $16.3 \%$ & 27.9 & $3.3 \%$ \\
\hline $2 \mathrm{~h}$ & 37.8 & 36.8 & $2.7 \%$ & 35.6 & $-3.5 \%$ \\
\hline $2 i$ & 46.9 & 44.4 & $5.3 \%$ & 48.3 & $8.1 \%$ \\
\hline $2 j$ & 30.5 & 26.8 & $12.0 \%$ & 24.9 & $-7.5 \%$ \\
\hline $2 \mathrm{k}$ & 44.4 & 39.1 & $11.8 \%$ & 42.5 & $8.0 \%$ \\
\hline $3 a$ & 39.2 & 25.3 & $35.4 \%$ & 30.5 & $16.8 \%$ \\
\hline $3 b$ & 42.0 & 29.3 & $30.4 \%$ & 35.6 & $17.8 \%$ \\
\hline $3 c$ & 38.9 & 31.7 & $18.4 \%$ & 34.5 & $8.0 \%$ \\
\hline $3 d$ & 44.3 & 40.0 & $9.9 \%$ & 38.6 & $-3.5 \%$ \\
\hline $3 e$ & 48.1 & 43.7 & $9.3 \%$ & 43.3 & $-0.9 \%$ \\
\hline 4 & 20.6 & 20.9 & $-1.4 \%$ & 22.6 & $7.3 \%$ \\
\hline
\end{tabular}

The simulation results for case 3 show that the average delay is better with the proposed FLTSC than the VAC-EP, in all cases, apart from cases $3 \mathrm{~d}$ and $3 \mathrm{e}$. In case 3, apart from cases $3 \mathrm{~d}$ and $3 \mathrm{e}$, the proposed FLTSC produces an improvement of 8.0-17.8\% in the average delay against the VAC-EP. In cases $3 \mathrm{~d}$ and $3 \mathrm{e}$, the proposed FLTSC show $3.5 \%$ and $0.9 \%$ increase in the average delay, respectively against the VAC-EP. The simulation results for case 4 show that the proposed FLTSC outperforms the VAC-EP. The average delay saving by the proposed FLTSC is $7.3 \%$ against the VAC-EP.

The proposed FLTSC has the ability to adjust the length of green time in response to real traffic flows variations. It performance depends on the traffic variations. The simulation results indicate that the FTC, VACEP and proposed FLTSC generally produce little difference in results when used in cases with constant traffic flows, whereas in cases of time-varying traffic the proposed FLTSC is superior to the FTC and VAC-EP.

The performance of the proposed FLTSC reduced as the traffic flows increased. In heavy flows or saturated conditions, the performance of the proposed FLTSC tends to close to the FTC and VAC-EP (cases 2 and 3). This is because in heavy flows, the length of green time of the proposed FLTSC had already reached its maximum value. As a result, the proposed FLTSC could not extend its green time to release more vehicle queueing on the approach. This led to longer delay.

\section{Conclusion}

This research was a pioneering effort in applying fuzzy logic to an ATS control for an isolated fourway intersection suitable for mixed traffic in developing countries, including a high proportion of motorcycles. Video image processing was used to capture maximum queue length (in meters) and average occupancy rate (in $\%$ ) from each approach of the intersection as an input data for the proposed FLTSC. The proposed FLTSC uses 
input data collected during the previous cycle, in order to estimate the length of green time required by each stage during the next cycle. This enables the framework signal plan to be adapted on a cycle-by-cycle basis.

The effectiveness of the FTC, VAC-EP and proposed FLTSC were analysed using VISSIM model in various traffic conditions, comparing average delay of vehicles at an intersection. In this research, to simulate mixed traffic conditions, all vehicles were modelled individually. By doing so, the effect of motorcycle behaviour to the performance of the VAC-EP can be analysed. This relates to the choice of the extension time that suitable for mixed traffic conditions, with high proportion of motorcycles. The simulation results indicate that the performance of the proposed FLTSC is generally better than the FTC and VAC-EP, especially in cases of time-varying traffic.

\section{Acknowledgements}

This research is sponsored by the University of Sebelas Maret Surakarta Indonesia. The author would also to thank to Prof. M.G.H. Bell (Imperial College United Kingdom) and S. Grosso (PTV) for their guidance and suggestion for this research.

\section{References}

[1] Strobel, H, Computer Controlled Urban Transport, Ed. John Wiley, 1982

[2] Kell, J.H. and Fullerton, I.J., Manual of traffic signal design - Chapter 7: Detectors. Institute of Transportation Engineers, Prentice Hall, Englewood Cliffs, New Jersey, 1991.

[3] Lee, J.H., Lee, K.M. and Leekwang, H., Fuzzy controller for intersection group. International IEEE/IAS Conference on Industrial Automation and Control, Taipei, Taiwan, 1994, pp. 376-382.

[4] Trabia, M.B. and Kaseko, M.S., A fuzzy logic controller for a traffic signal. IASTED International Conference on Applications of Control and Robotics, Orlando, Florida, January 1996, pp. 117-122

[5] Kim, J., A fuzzy logic control simulator for adaptive traffic management, IEEE International Conference on Fuzzy Systems, vol. 3, 1997, pp. 1519-1524.

[6] Bell, M.G.H., Future directions in traffic signal control. Transportation Research Part A, Vol. 26A, No. 4, 1992, pp. 303-313.

[7] Trabia, M.B., Kaseko, M.S. and Ande, M., A two-stage fuzzy logic for traffic signals. Transportation Research Part C, Vol. 7, No. 7, 1999, pp. 353-367.

[8] Bång, K.L., Optimal control of isolated traffic signals. Traffic Engineering and Control, Vol. 17, No. 7, July, 1976, pp. $288-292$.

[9] Vincent, R.A. and Young, C.P., Self-optimising traffic signal control using microprocessors - the TRRL MOVA strategy for isolated intersections. Traffic Engineering and Control, Vol. 27, No. 7, 1986, pp. 385-387.

[10] Zadeh, L.A., Fuzzy sets. Information and Control, Vol. 8, 1965, pp. 338-353.

[11] Pappis, C.P. and Mamdani, E.H., A fuzzy controller for a traffic junction. IEEE Transactions on Systems, Man, and Cybernetics, Vol. SMC-7, No. 10, 1977, pp. 707-717.

[12] Kou, K.Y., A fuzzy model of change and clearance intervals at a signalised intersection. PhD Thesis, The University of Texas at Arlington, USA, 1995.

[13] Niittymaki, J. and Pursula, M., Signal control using fuzzy logic. Fuzzy Sets and System, 116, 2000, pp. 11-22.

[14] Sayers, T.M., Anderson, J. and Clement, S., The multi-objective optimisation of a traffic control system. Abbreviated Presentation Sessions of the $14^{\text {th }}$ International Symposium on Transportation and Traffic Theory. Jerusalem, Israel, 20-23 July 1999, pp. 153176.

[15] Indonesian Highway Capacity Manual, Directorate General Bina Marga Indonesia, 1997.

[16] Webster, F., Traffic signal settings. Road Research Tehnical Paper No. 39, London, 1958.

[17] Kell, J.H and Fullerton, I J (1991) Manual of Traffic Signal Design - Chapter 7: Detectors, Institute of Transportation Engineers, Prentice Hall, Englewood Cliffs, New Jersey.

[18] Sayers, T.M., Bell, M.G.H., Meiden, T. and Busch, F., Traffic responsive signal control using fuzzy logic - a practical modular approach. Proceedings EUFIT 1996, Aachen, Germany, September 2-4, 1996, pp. 2159-2163.

[19] Sutomo, H., Appropriate saturation flow at traffic signals in Javanese cities: a modelling approach. PhD Thesis, Institute for Transport Studies, University of Leeds, UK, 1992.

[20] Lee, C.C., Fuzzy logic in control systems: fuzzy logic controller - Part I. IEEE Transactions on Systems, Man, and Cybernetics, Vol. 20, No. 2, March/April, 1990, pp. 404-418.

[21] Kim, S., Applications of petri networks and fuzzy logic to advanced traffic management systems. PhD Thesis, Polytechnic University, USA, 1994.

[22] Driankov, D., Hellendoorn, H. and Reinfrank, M., An introduction to fuzzy logic. Second Edition, Springer-Verlag Berlin Heidelberg, 1996.

[23] Mamdani, E.H. and Assilian, S., An experiment in linguistic synthesis with a fuzzy controller. International Journal of ManMachine Studies, Vol. 7, No. 1, 1975, pp. 1-13.

[24] Yulianto, B., Application of fuzzy logic to traffic signal control under mixed traffic conditions. Traffic Engineering and Control, International Journal of Traffic Management and Transportation Planning, Vol. 44, No. 9, November, 2003, pp. 332-336.

[25] Yulianto, B., Performance of Vehicle Actuated Control under Mixed Traffic Conditions, Jurnal Gema Teknik, Majalah Ilmiah Teknik, Fakultas Teknik Universitas Sebelas Maret, Indonesia, Nomor 2/Tahun X, pp. 7-15, 2007, Juli.

[26] Roess, R.P., McShane, W.R. and Prassas, E.S., Traffic Engineering. Prentice-Hall Inc., New Jersey, 1998. 